\title{
ON THE FOURIER EXPANSION OF WORD MAPS
}

\author{
ORI PARZANCHEVSKI AND GILI SCHUL
}

\begin{abstract}
Frobenius observed that the number of times an element of a finite group is obtained as a commutator is given by a specific combination of the irreducible characters of the group. More generally, for any word $w$ the number of times an element is obtained by substitution in $w$ is a class function. Thus, it has a presentation as a combination of irreducible characters, called its Fourier expansion. In this paper we present formulas regarding the Fourier expansion of words in which some letters appear twice. These formulas give simple proofs for classical results, as well as new ones.
\end{abstract}

\section{INTRODUCTION}

Let $w$ be a word in $\mathbf{F}_{d}=\mathbf{F}\left(x_{1}, \ldots, x_{d}\right)$, the free group on $d$ generators. Given a finite group $G, w$ induces a word map from $G^{d}=G \times \cdots \times G$ to $G$, defined by substitution: if $w=\prod_{j=1}^{r} x_{i_{j}}^{\varepsilon_{j}}$ (where $\varepsilon_{j}= \pm 1$ ), then the word map $w: G^{d} \rightarrow G$ is defined by $w\left(g_{1}, \ldots, g_{d}\right)=\prod_{j=1}^{r} g_{i_{j}}^{\varepsilon j}$. For example, for $w=\left[x_{1}, x_{2}\right]=x_{1} x_{2} x_{1}^{-1} x_{2}^{-1}, w(g, h)=g h g^{-1} h^{-1}$. It is a classical question to understand the distribution which $w$ induces on $G$, i.e., the function

$$
N_{w}(g)=\left|w^{-1}(g)\right|=\sharp\left\{\left(g_{1}, \ldots, g_{d}\right) \in G^{d} \mid w\left(g_{1}, \ldots, g_{d}\right)=g\right\}
$$

(some authors write $N_{w, G}$ to emphasis that this depends on $G$ ). The simple observation that $N_{w}$ is a class function shows that one can write

$$
N_{w}=\sum_{\chi \in \operatorname{Irr}(G)} N_{w}^{\chi} \cdot \chi
$$

for unique $N_{w}^{\chi} \in \mathbb{C}$, where $\operatorname{Irr}(G)$ are the irreducible characters of $G$. The coefficients $N_{w}^{\chi}$ are called the Fourier coefficients of $w$ with respect to $G$, and their study goes back to Frobenius [Fro96, who showed that $N_{\left[x_{1}, x_{2}\right]}^{\chi}=\frac{|G|}{\chi(1)}$ for any $G$. Since $\chi(1)$ divides $|G|$, this shows in particular that $N_{\left[x_{1}, x_{2}\right]}$ is itself a character of $G$. Several authors have noticed that Frobenius' argument generalizes to $N_{\left[x_{1}, y_{1}\right] \ldots \cdot\left[x_{k}, y_{k}\right]}^{\chi}=\left(\frac{|G|}{\chi(1)}\right)^{2 k-1}$, and the Fourier expansions of other words were studied as well (see [Isa94, chapter 4], and more recent results in [Tam00, AV11]).

If $w$ and $w^{\prime}$ are equivalent under Aut $\left(\mathbf{F}_{d}\right)$, then $N_{w} \equiv N_{w^{\prime}}$ for any $G$, so that one can always replace $w \in \mathbf{F}_{d}$ by $\varphi(w)$ for any $\varphi \in \operatorname{Aut}\left(\mathbf{F}_{d}\right)$. If some letter $x$ appears only once in $w$ (either as $x$ or as $x^{-1}$, but not both), then $w$ is equivalent under Aut $\left(\mathbf{F}_{d}\right)$ to the single letter $x$, and thus $N_{w}$ is constant. In this paper we consider the next simplest case, in which some letter appears twice in $w$, either with the same sign or with different signs.

We say that $x$ is a square in $w$ if $x$ appears twice in it, and $x^{-1}$ does not, or vice versa. We say that $x$ is dismissible in $w$ if both $x$ and $x^{-1}$ appear in $w$, each of them only once. In this paper we present formulas regarding the Fourier coefficients of words with at least one square 
or dismissible letter. The results in [Fro96, Tam00, AV11] are obtained as special cases of these formulas, and further cases and applications are shown in Section 2 .

1.1. Statement of the results. It is well known that for any word $w \in \mathbf{F}_{d}$ and finite group $G$, the Fourier coefficients of $w$ with respect to $G$ can be obtained by summation over all substitutions of $G$ 's elements in $w$ 's letters:

$$
N_{w}^{\chi}=\frac{1}{|G|} \sum_{\bar{x} \in G^{d}} \bar{\chi}\left(w\left(x_{1}, \ldots, x_{d}\right)\right)
$$

where $\bar{x}$ stands for $\left(x_{1}, \ldots, x_{d}\right)$ (for the proof see Proposition 1). We will show the following:

Theorem. Let $w$ be a word in $\mathbf{F}_{d}, G$ a finite group, and $\chi \in \operatorname{Irr}(G)$.

(1) There is a formula for $N_{w}^{\chi}$ which involves summation only over the non-dismissible letters of $w$.

(2) There is a formula for $N_{w}^{\chi}$ which involves the Frobenius-Schur indicator of $\chi$ (1.2), and summation only over the non-dismissible and non-square letters of $w$.

The Frobenius-Schur indicator of $\chi$ is

$$
\mathcal{F S}_{\chi}= \begin{cases}1 & \chi \text { is afforded by a real representation } \\ -1 & \chi \text { is real but not afforded by a real representation } \\ 0 & \chi \text { is not real, }\end{cases}
$$

and it is a classical fact that $\mathcal{F} \mathcal{S}_{\chi}=N_{x^{2}}^{\chi}$ (see e.g. [Ser77, Proposition 39]).

The formulas obtained are the following:

(1) Dismissible letters: Let $w \in \mathbf{F}\left(x_{1}, \ldots, x_{d}, y_{1}, \ldots, y_{n}\right)$ be a word in which $y_{1}, \ldots, y_{n}$ are dismissible letters. Define words $w_{0}, \ldots, w_{2 n} \in \mathbf{F}\left(x_{1}, \ldots, x_{d}\right)$ by writing $w$ as

$$
w=w_{0} \cdot y_{i_{1}}^{ \pm 1} \cdot w_{1} \cdot y_{i_{2}}^{ \pm 1} \cdot \ldots \cdot w_{2 n-1} \cdot y_{i_{n}}^{ \pm 1} \cdot w_{2 n},
$$

where any $w_{j}$ may be the empty word 1 . We define words $W_{i} \in \mathbf{F}\left(x_{1}, \ldots, x_{d}\right)$ as follows. For $W_{1}$, start reading $w$ from $w_{0}$, and upon reaching a dismissible letter, jump to its inverse, and continue reading from there. When reaching the end of $w$, stop reading the word obtained so far is $W_{1}$. Now, find the first $w_{j}$ which has not been read yet, and start reading $W_{2}$ from it, again jumping from dismissible letters to their inverse. You finish reading $W_{2}$ upon reaching your starting point. Continuing in this manner, one obtains $W_{1}, \ldots, W_{r}$ (where $r=r(w)$ ) - the process stops when all $w_{j}$ have been read. Our formula then states that the Fourier expansion of $w$ is given by

$$
N_{w}^{\chi}=\frac{|G|^{n-1}}{\chi(1)^{n}} \sum_{\bar{x} \in G^{d}} \bar{\chi}\left(W_{1}(\bar{x})\right) \cdot \ldots \cdot \bar{\chi}\left(W_{r}(\bar{x})\right) .
$$

It may happen that $d=0$ (if all letters are dismissible), in which case the summation is over the empty substitution, and

$$
N_{w}^{\chi}=\frac{|G|^{n-1}}{\chi(1)^{n-r}} .
$$


(2) Squares: Let $w=w_{1} \cdot y \cdot w_{2} \cdot y \cdot w_{3} \in \mathbf{F}\left(x_{1}, \ldots, x_{d}, y\right)$ where $y$ does not appear in $w_{1}, w_{2}, w_{3} \in$ $\mathbf{F}\left(x_{1}, \ldots, x_{d}\right)$. Then

$$
N_{w}^{\chi}=\frac{|G|}{\chi(1)} \mathcal{F} \mathcal{S}_{\chi} N_{w_{1} w_{2}^{-1} w_{3}}^{\chi},
$$

where in $N_{w_{1} w_{2}^{-1} w_{3}}^{\chi}$ we refer to $w_{1} w_{2}^{-1} w_{3}$ as a word in $\mathbf{F}\left(x_{1}, \ldots, x_{d}\right)$.

Example. To demonstrate the formula for dismissible letters, let

$$
w=\underbrace{x_{1}}_{w_{0}} y_{1} \underbrace{x_{1} x_{2}}_{w_{1}} y_{3} \underbrace{x_{2} x_{1}}_{w_{2}} y_{1}^{-1} \underbrace{x_{1}^{3}}_{w_{3}} y_{2} \underbrace{x_{3}^{-1}}_{w_{4}} y_{3}^{-1} \underbrace{x_{3}^{2}}_{w_{5}} y_{2}^{-1} \underbrace{x_{3}}_{w_{6}} .
$$

We obtain

$$
\begin{aligned}
& W_{1}=w_{0} w_{3} w_{6}=x_{1}^{4} x_{3} \\
& W_{2}=w_{1} w_{5} w_{4} w_{2}=x_{1} x_{2} x_{3} x_{2} x_{1} .
\end{aligned}
$$

and thus

$$
N_{w}^{\chi}=\frac{|G|^{2}}{\chi(1)^{3}} \sum_{x_{1}, x_{2}, x_{3} \in G} \bar{\chi}\left(x_{1}^{4} x_{3}\right) \bar{\chi}\left(x_{1} x_{2} x_{3} x_{2} x_{1}\right) .
$$

In the next section we demonstrate some applications of the formulas, including the results referred to earlier. Section 3 presents some preparatory results for the main theorems, which are proved in Section 4

Remarks.

(1) The ambient free group of $w$ affects $N_{w}$. For example, for $w=x y \in F(x, y)$ we have $N_{w} \equiv|G|$ (for every finite $G$ ), but for $w=x y \in F(x, y, z)$ we have $N_{w} \equiv|G|^{2}$.

(2) For 1 the empty word on zero letters, we have $N_{1}(g)=\delta_{g, e}$ and thus $N_{1}^{\chi}=\frac{\chi(1)}{|G|}$.

(3) In order to keep things tidy we shall occasionally write $w$ for $w\left(x_{1}, \ldots, x_{d}\right)$, where $x_{1}, \ldots, x_{d}$ are elements of $G$. For example, we write (1.3) as

$$
N_{w}^{\chi}=\frac{|G|^{n-1}}{\chi(1)^{n}} \sum_{\bar{x} \in G^{d}} \bar{\chi}\left(W_{1}\right) \cdot \ldots \cdot \bar{\chi}\left(W_{r}\right) .
$$

Acknowledgements. We would like to thank our advisors Aner Shalev and Alex Lubotzky for their support, and Doron Puder and Zlil Sela for valuable discussions. This research was supported by an ERC Advanced Grant.

\section{Corollaries and applications}

We give now examples of applications and corollaries of the main formulas.

(1) Frobenius' formula: for the commutator

$$
w=\left[y_{1}, y_{2}\right]=y_{1} y_{2} y_{1}^{-1} y_{2}^{-1}=w_{0} y_{1} w_{1} y_{2} w_{2} y_{1}^{-1} w_{3} y_{2}^{-1} w_{4}
$$

we have $d=0, n=2$, and $w_{0}, \ldots, w_{4}=1$. This gives $W_{1}=w_{0} w_{3} w_{2} w_{1} w_{4}=1$, so that $r=1$, and thus by (1.4)

$$
N_{\left[y_{1}, y_{2}\right]}^{\chi}=\frac{|G|}{\chi(1)} .
$$


More generally, $w=\left[y_{1}, y_{2}\right] \cdot \ldots \cdot\left[y_{2 k-1}, y_{2 k}\right]$ gives $d=0, n=2 k, r=1$, and $W_{1}=1$, hence

$$
N_{\left[y_{1}, y_{2}\right] \cdots \cdot\left[y_{2 k-1}, y_{2 k}\right]}^{\chi}=\left(\frac{|G|}{\chi(1)}\right)^{2 k-1}
$$

(which can also be deduced from (3.1)).

(2) Let $w_{0}, w_{1}$ be words in $\mathbf{F}\left(x_{1}, \ldots, x_{d}\right)$ (not necessarily disjoint), and observe $w=w_{0} y w_{1} y^{-1}$. In this case $W_{1}=w_{0}$ and $W_{2}=w_{1}$, which gives

$$
N_{w_{0} y w_{1} y^{-1}}^{\chi}=\frac{1}{\chi(1)} \sum_{\bar{x} \in G^{d}} \bar{\chi}\left(w_{0}\right) \bar{\chi}\left(w_{1}\right) .
$$

(3) For $w \in \mathbf{F}_{d}$, taking $w_{0}=w, w_{1}=w^{-1}$ in the previous example gives

$$
\begin{aligned}
N_{[w, y]}^{\chi} & =\frac{1}{\chi(1)} \sum_{\bar{x} \in G^{d}} \bar{\chi}(w) \chi(w)=\frac{1}{|\chi(1)|} \sum_{g \in G} N_{w}(g) \bar{\chi}(g) \chi(g) \\
& =\frac{|G|}{\chi(1)}\left\langle N_{w} \chi, \chi\right\rangle .
\end{aligned}
$$

In terms of the Fourier expansion of $N_{w}$, we obtain

$$
N_{[w, y]}^{\chi}=\frac{|G|}{\chi(1)} \sum_{\psi \in \operatorname{Irr}(G)}\langle\psi \chi, \chi\rangle N_{w}^{\psi},
$$

which is (roughly) Lemma 4.6 in AV11. We shall return to this example in Proposition 2.

(4) Similarly, for $w_{0}=w_{1}=w$ we obtain

$$
N_{w y w y^{-1}}^{\chi}=\frac{1}{|\chi(1)|} \sum_{g \in G} N_{w}(g) \chi(g)^{2}=\frac{|G|}{\chi(1)}\left\langle N_{w} \chi, \bar{\chi}\right\rangle .
$$

(5) If $W_{1}, \ldots, W_{r}=1$, then

$$
N_{w}^{\chi}=\frac{|G|^{n-1}}{\chi(1)^{n}} \sum_{\bar{x} \in G^{d}} \bar{\chi}\left(W_{1}\right) \cdot \ldots \cdot \bar{\chi}\left(W_{r}\right)=\frac{|G|^{n+d-1}}{\chi(1)^{n-r}}
$$

and the only question is what is $r$. In this case one can show that $w$ is equivalent under Aut $\left(\mathbf{F}_{d}\right)$ to a word $w^{\prime}$ in which all letters are dismissible (such a word is called admissible). It is a classical observation that admissible words describe a relation defining the fundamental group of an orientable surface. Thus, $w^{\prime}$ is equivalent under Aut $\left(\mathbf{F}_{d}\right)$ to a product of $g$ disjoint commutators, where $g$ is the genus of the surface it defines. Finding $r$ amounts to finding this genus, as $g=\frac{n-r+1}{2}$.

(6) For example, for the admissible words $w=y_{1} y_{2} \ldots y_{n} y_{1}^{-1} y_{2}^{-1} \ldots y_{n}^{-1}$ we obtain a result from [Tam00]: for $n$ odd we have

$$
\begin{aligned}
& W_{1}=w_{0} w_{n+1} w_{2} w_{n+3} w_{4} \ldots w_{n-1} w_{2 n}=1 \\
& W_{2}=w_{1} w_{n+2} w_{3} w_{n+4} \ldots w_{n-2} w_{2 n-1} w_{n}=1
\end{aligned}
$$

so that $r=2$ and $N_{w}^{\chi}=\frac{|G|^{n-1}}{\chi(1)^{n-2}}$. For $n$ even,

$$
W_{1}=w_{0} w_{n+1} w_{2} w_{n+3} w_{4} \ldots w_{2 n-1} w_{n} w_{1} w_{n+2} w_{3} w_{n+4} \ldots w_{n-2} w_{2 n-1} w_{n}=1
$$


covers all $w_{i}$, hence $r=1$ and $N_{w}^{\chi}=\frac{|G|^{n-1}}{\chi(1)^{n-1}}$.

(7) Combining (3) and (11) gives a formula for $w=[[x, y], z]$ :

$$
N_{[[x, y], z]}^{\chi}=\frac{|G|}{\chi(1)} \sum_{\psi \in \operatorname{Irr}(G)}\langle\psi \chi, \chi\rangle N_{[x, y]}^{\psi}=\frac{|G|^{2}}{\chi(1)} \sum_{\psi \in \operatorname{Irr}(G)} \frac{\langle\psi \chi, \chi\rangle}{\psi(1)} .
$$

This formula was already obtained, using different methods, by Amit \& Vishne AV11. In particular it implies that knowing the decomposition of tensor product of irreducible representation of $G$ gives the Fourier expansion of $[[x, y], z] 1]$ Continuing by induction gives the coefficients of $\left[\left[\left[x_{1}, x_{2}\right], x_{3}\right], \ldots, x_{n}\right]$, which can be presented in terms of matrix exponentiation (see AV11]).

(8) Nested and non-nested squares: if $w=w_{1} \cdot x \cdot w_{2} \cdot x \cdot w_{3} \cdot y \cdot w_{4} \cdot y \cdot w_{5}$ where the $w_{i}$ do not contain $x$ and $y$ (but are not necessarily disjoint), then

$$
\begin{aligned}
N_{w}^{\chi} & =\frac{|G|}{\chi(1)} \cdot \mathcal{F} \mathcal{S}_{\chi} \cdot N_{w_{1} x w_{2} x w_{3} w_{4}^{-1} w_{5}}^{\chi}=\frac{|G|^{2}}{\chi(1)^{2}} \cdot \mathcal{F} \mathcal{S}_{\chi}^{2} \cdot N_{w_{1} w_{2}^{-1} w_{3} w_{4}^{-1} w_{5}}^{\chi} \\
& =\frac{|G|^{2}}{\chi(1)^{2}} \cdot \delta_{\chi \in \mathbb{R}} \cdot N_{w_{1} w_{2}^{-1} w_{3} w_{4}^{-1} w_{5}}^{\chi}
\end{aligned}
$$

where $\delta_{\chi \in \mathbb{R}}$ is one if $\chi$ is real and zero otherwise. For $w^{\prime}=w_{1} \cdot x \cdot w_{2} \cdot y \cdot w_{3} \cdot x \cdot w_{4} \cdot y \cdot w_{5}$, however, we obtain

$$
N_{w^{\prime}}^{\chi}=\frac{|G|}{\chi(1)} \cdot \mathcal{F} \mathcal{S}_{\chi} \cdot N_{w_{1} x w_{2} w_{4}^{-1} x^{-1} w_{3}^{-1} w_{5}}^{\chi}
$$

in which $x$ became dismissible, and by (1.3)

$$
N_{w^{\prime}}^{\chi}=\frac{|G|}{\chi(1)^{2}} \cdot \mathcal{F} \mathcal{S}_{\chi} \sum_{\bar{x} \in G^{d}} \bar{\chi}\left(w_{1} w_{3}^{-1} w_{5}\right) \bar{\chi}\left(w_{2} w_{4}^{-1}\right) .
$$

Had we taken $w^{\prime \prime}=w_{1} \cdot x \cdot w_{2} \cdot y \cdot w_{3} \cdot x^{-1} \cdot w_{4} \cdot y \cdot w_{5}$, we would get

$$
N_{w^{\prime \prime}}^{\chi}=\frac{|G|}{\chi(1)} \cdot \mathcal{F} \mathcal{S}_{\chi} \cdot N_{w_{1} x w_{2} w_{4}^{-1} x w_{3}^{-1} w_{5}}^{\chi},
$$

i.e. $x$ became a square, and now

$$
N_{w^{\prime \prime}}^{\chi}=\frac{|G|^{2}}{\chi(1)^{2}} \cdot \delta_{\chi \in \mathbb{R}} \cdot N_{w_{1} w_{4} w_{2}^{-1} w_{3}^{-1} w_{5}}^{\chi} .
$$

Notice that had we applied (1.3) to $w^{\prime \prime}$, we would have get

$$
N_{w^{\prime \prime}}^{\chi}=\frac{1}{\chi(1)} \sum_{\substack{x \in G^{d} \\ y \in G}} \bar{\chi}\left(w_{1} w_{4} y w_{5}\right) \bar{\chi}\left(w_{2} y w_{3}\right)
$$

which requires summation over more substitutions then (2.4). In general, squares are better taken care of before dismissible letters.

\footnotetext{
${ }^{1}$ If this decomposition is given by $\chi_{i} \cdot \chi_{j}=\sum c_{i, j, k} \cdot \chi_{k}$, then $c_{i, j, k}$ are the Clebsch-Gordan coefficients of $G$. For $G=S_{n}$ they are also referred to as Kronecker coefficients, and for $G=\mathrm{GL}_{n}$ as Littlewood-Richardson coefficients.
} 
(9) Denote $\{x, y\} \stackrel{\text { def }}{=} x y x y^{-1}$. By (2.2), or by (2.4), we have $N_{\{x, y\}}^{\chi}=\frac{|G|}{\chi(1)} \cdot \delta_{\chi \in \mathbb{R}}$. Comparing this with $N_{[x, y]}^{\chi}=\frac{|G|}{\chi(1)}$ we see that $N_{\{x, y\}} \equiv N_{[x, y]}$ iff all character of $G$ are real. This happens iff every element of $g$ is conjugate to its inverse, and it is an easy exercise that this in itself is equivalent to $N_{\{x, y\}} \equiv N_{[x, y]}$. However, when considering $w=$ $\prod_{i=1}^{k}\left[x_{i}, y_{i}\right]$ and $w^{\prime}=\prod_{i=1}^{k}\left\{x_{i}, y_{i}\right\}$, we have

$$
N_{w}^{\chi}=\left(\frac{|G|}{\chi(1)}\right)^{2 k-1} \quad \text { and } \quad N_{w^{\prime}}^{\chi}=\left(\frac{|G|}{\chi(1)}\right)^{2 k-1} \cdot \delta_{\chi \in \mathbb{R}}
$$

(either by computing directly or by (3.1)). This shows that once more $N_{w} \equiv N_{w^{\prime}}$ iff all characters are real, and this does not seem to be as simple to show directly.

(10) Similarly, applying (1.3) to $w=[a, b] d[a, c] d^{-1}$ and $w^{\prime}=\{a, b\} d\{a, c\} d^{-1}$ gives

$$
N_{w}^{\chi}=\frac{|G|^{2}}{\chi(1)^{3}} \sum_{g \in G}|\chi(g)|^{4}, \quad N_{w^{\prime}}^{\chi}=\frac{|G|^{2}}{\chi(1)^{3}} \sum_{g \in G}(\chi(g))^{4}
$$

which shows that $N_{w} \equiv N_{w^{\prime}}$ iff $\chi(g) \in \mathbb{R} \cup i \mathbb{R}$ for every $\chi \in \operatorname{Irr}(G)$ and $g \in G$. We leave it as an exercise to find, for any $k \in \mathbb{N}$, words $w, w^{\prime}$ which induce the same distribution on $G$ iff $\chi(g)^{k} \in \mathbb{R}$ for all $\chi \in \operatorname{Irr}(G)$ and $g \in G$.

(11) For a word with dismissible letters for which $r(w)=1$, we obtain

$$
N_{w}^{\chi}=\frac{|G|^{n-1}}{\chi(1)^{n}} \sum_{\bar{x} \in G^{d}} \bar{\chi}\left(W_{1}\right)=\frac{|G|^{n}}{\chi(1)^{n}} N_{W_{1}}^{\chi} .
$$

Since $r=1, n$ is even (see Theorem 4), and we obtain by (3.1)

$$
N_{w}^{\chi}=N_{\left[y_{1}, y_{2}\right] \ldots \cdot\left[y_{n-1}, y_{n}\right] \cdot W_{1}}^{\chi} .
$$

In fact, one can show that $w$ and $\left[y_{1}, y_{2}\right] \cdot \ldots \cdot\left[y_{n-1}, y_{n}\right] \cdot W_{1}$ are equivalent under $\operatorname{Aut}\left(\mathbf{F}_{d}\right)$.

(12) More generally, the formula (1.3) can be interpreted in the context of tuples of word maps, as follows: for $w_{1}, \ldots, w_{r} \in \mathbf{F}_{d}$, we observe the tuple $\overrightarrow{\boldsymbol{w}}=\left(w_{1}, \ldots, w_{r}\right)$ as a word map $\overrightarrow{\boldsymbol{w}}: G^{d} \rightarrow G^{r}$, defined by

$$
\overrightarrow{\boldsymbol{w}}\left(x_{1}, \ldots, x_{d}\right)=\left(w_{1}(\bar{x}), \ldots, w_{r}(\bar{x})\right),
$$

and define

$$
N_{\overrightarrow{\boldsymbol{w}}}\left(g_{1}, \ldots, g_{r}\right)=\left|(\overrightarrow{\boldsymbol{w}})^{-1}(\bar{g})\right|=\sharp\left\{\bar{x} \in G^{d} \mid \overrightarrow{\boldsymbol{w}}(\bar{x})=\bar{g}\right\} .
$$

Though $N_{\overrightarrow{\boldsymbol{w}}}$ is not a class function on $G^{r}$, we can still observe the "Fourier coefficient" of $\eta \in \operatorname{Irr}\left(G^{r}\right)$,

$$
N_{\overrightarrow{\boldsymbol{w}}}^{\eta}=\left\langle N_{\overrightarrow{\boldsymbol{w}}}, \eta\right\rangle=\frac{1}{|G|^{r}} \sum_{\bar{g} \in G^{r}} N_{\overrightarrow{\boldsymbol{w}}}(\bar{g}) \bar{\eta}(\bar{g})
$$


With this notation we find that if the dismissible letter formula for a word $w$ produces $W_{1}, \ldots, W_{r}$, then for every $\chi \in \operatorname{Irr}(G)$

$$
\begin{aligned}
N_{w}^{\chi} & =\frac{|G|^{n-1}}{\chi(1)^{n}} \sum_{\bar{x} \in G^{d}} \bar{\chi}\left(W_{1}(\bar{x})\right) \cdot \ldots \cdot \bar{\chi}\left(W_{r}(\bar{x})\right) \\
& =\frac{|G|^{n+r-1}}{\chi(1)^{n}} \cdot \frac{1}{|G|^{r}} \sum_{\bar{g} \in G^{r}} N_{\overrightarrow{\mathbf{W}}}(\bar{g}) \cdot \bar{\chi}\left(g_{1}\right) \cdot \ldots \cdot \bar{\chi}\left(g_{r}\right) \\
& =\frac{|G|^{n+r-1}}{\chi(1)^{n}}\left\langle N_{\overrightarrow{\mathbf{W}}}, \chi^{\otimes r}\right\rangle \\
& =\frac{|G|^{n+r-1}}{\chi(1)^{n}} N_{\overrightarrow{\mathbf{W}}}^{\chi^{\otimes r}}
\end{aligned}
$$

where $\overrightarrow{\mathbf{W}}=\left(W_{1}, \ldots, W_{r}\right)$.

\section{Preliminaries}

The following proposition lists some simple facts about the Fourier expansions of word maps.

Proposition 1. Let $G$ be a finite group, $w \in \mathbf{F}_{d}$, and $\chi \in \operatorname{Irr}(G)$.

(1) The coefficient of $\chi$ in $N_{w}$ is given by

$$
N_{w}^{\chi}=\frac{1}{|G|} \sum_{\bar{x} \in G^{d}} \bar{\chi}(w)=\frac{1}{|G|} \sum_{\bar{x} \in G^{d}} \bar{\chi}\left(w\left(x_{1}, \ldots, x_{d}\right)\right) .
$$

(2) The Fourier expansion of $N_{w^{-1}}$ is given by $N_{w^{-1}}^{\chi}=\overline{N_{w}^{\chi}}$.

(3) If $w_{1}, w_{2} \in \mathbf{F}_{d}$ are words with disjoint letters then

$$
N_{w_{1} \cdot w_{2}}^{\chi}=\frac{|G|}{\chi(1)} N_{w_{1}}^{\chi} N_{w_{2}}^{\chi}
$$

Proof.

(1) It is well known that $\operatorname{Irr}(G)$ is an orthonormal base for the space of class functions on $G$, endowed with inner product

$$
\left\langle f_{1}, f_{2}\right\rangle=\frac{1}{|G|} \sum_{g \in G} f_{1}(g) \overline{f_{2}(g)}
$$

Thus,

$$
\begin{aligned}
N_{w}^{\chi} & =\left\langle N_{w}, \chi\right\rangle=\frac{1}{|G|} \sum_{g \in G} N_{w}(g) \bar{\chi}(g) \\
& =\frac{1}{|G|} \sum_{g \in G} \sum_{\substack{\bar{x} \in G^{d} \text { s.t. } \\
w\left(x_{1}, \ldots, x_{d}\right)=g}} \bar{\chi}(g)=\frac{1}{|G|} \sum_{\bar{x} \in G^{d}} \bar{\chi}\left(w\left(x_{1}, \ldots, x_{d}\right)\right) .
\end{aligned}
$$

(2) This follows from

$$
N_{w^{-1}}(g)=\overline{N_{w^{-1}}(g)}=\overline{N_{w}\left(g^{-1}\right)}=\sum_{\chi \in \operatorname{Irr}(G)} \overline{N_{w}^{\chi} \chi\left(g^{-1}\right)}=\sum_{\chi \in \operatorname{Irr}(G)} \overline{N_{w}^{\chi} \chi}(g) .
$$


(3) We recall that the convolution of two class functions is defined by

$$
\left(f_{1} * f_{2}\right)(g)=\frac{1}{|G|} \sum_{h \in G} f_{1}(h) f_{2}\left(h^{-1} g\right)
$$

and that for $\chi, \psi \in \operatorname{Irr}(G)$

$$
\psi * \chi=\frac{\delta_{\psi, \chi}}{\chi(1)} \cdot \chi
$$

(see e.g. [Fol95, 5.20]). Using this, we see that

$$
\begin{aligned}
N_{w_{1} \cdot w_{2}}(g) & =\sum_{h \in G} N_{w_{1}}(h) N_{w_{2}}\left(h^{-1} g\right) \\
& =\sum_{h \in G}\left(\sum_{\chi \in \operatorname{Irr}(G)} N_{w_{1}}^{\chi} \cdot \chi(h)\right) \cdot\left(\sum_{\psi \in \operatorname{Irr}(G)} N_{w_{2}}^{\psi} \cdot \psi\left(h^{-1} g\right)\right) \\
& =\sum_{\chi, \psi \in \operatorname{Irr}(G)} N_{w_{1}}^{\chi} \cdot N_{w_{2}}^{\psi} \cdot\left(\sum_{h \in G} \chi(h) \psi\left(h^{-1} g\right)\right) \\
& =\sum_{\chi, \psi \in \operatorname{Irr}(G)} N_{w_{1}}^{\chi} \cdot N_{w_{2}}^{\psi} \cdot|G| \cdot(\chi * \psi)(g) \\
& =\sum_{\chi, \psi \in \operatorname{Irr}(G)} N_{w_{1}}^{\chi} \cdot N_{w_{2}}^{\psi} \cdot \frac{|G|}{\chi(1)} \cdot \delta_{\chi, \psi} \chi(g) \\
& =\sum_{\chi \in \operatorname{Irr}(G)} \frac{|G|}{\chi(1)} N_{w_{1}}^{\chi} N_{w_{2}}^{\chi} \chi(g) .
\end{aligned}
$$

The next proposition already appears in [AV11] (as Lemma 4.6), and it was also shown in (2.1) to follow from the formula for dismissible letters (1.3). Nevertheless, we present here two proofs for it. The first is interesting in its own rights, and the second will be generalized to the proof of the formula (1.3), and serves as a warm-up.

Proposition 2. If $y$ is a letter which does not appear in $w$, then

$$
N_{[w, y]}^{\chi}=\frac{|G|}{\chi(1)}\left\langle N_{w} \chi, \chi\right\rangle=\frac{|G|}{\chi(1)} \sum_{\psi \in \operatorname{Irr}(G)}\langle\psi \chi, \chi\rangle N_{w}^{\psi} .
$$

First proof. The column orthogonality of characters states that

$$
\sum_{\chi \in \operatorname{Irr}(G)} \chi(g) \bar{\chi}(h)=\left|C_{G}(g)\right| \delta_{g \sim h} \quad \text { where } \quad \delta_{g \sim h}=\left\{\begin{array}{ll}
1 & g^{G}=h^{G} \\
0 & \text { else }
\end{array} .\right.
$$


Thus,

$$
\begin{aligned}
N_{[w, y]}(g) & =\sum_{x \in G} N_{w}(x) \cdot \sharp\{y \in G \mid[x, y]=g\} \\
& =\sum_{x \in G} N_{w}(x) \cdot \sharp\left\{y \in G \mid y x^{-1} y^{-1}=x^{-1} g\right\} \\
& =\sum_{x \in G} N_{w}(x) \cdot\left|C_{G}\left(x^{-1}\right)\right| \cdot \delta_{x^{-1} \sim x^{-1} g} \\
& =\sum_{x \in G} N_{w}(x) \sum_{\chi \in \operatorname{Irr}(G)} \chi\left(x^{-1}\right) \bar{\chi}\left(x^{-1} g\right) \\
& =\sum_{x \in G} N_{w}(x) \sum_{\chi \in \operatorname{Irr}(G)} \bar{\chi}\left(x^{-1}\right) \chi\left(x^{-1} g\right) \\
& =\sum_{\chi \in \operatorname{Irr}(G)} \sum_{x \in G} N_{w}(x) \chi(x) \chi\left(x^{-1} g\right) \\
& =|G| \sum_{\chi \in \operatorname{Irr}(G)}\left(N_{w} \chi * \chi\right)(g) .
\end{aligned}
$$

By (3.2) and linearity, any class function $f$ and $\chi \in \operatorname{Irr}(G)$ satisfy

$$
f * \chi=\frac{\langle f, \chi\rangle}{\chi(1)} \chi
$$

and for $f=N_{w} \chi$ this gives

$$
N_{[w, y]}(g)=\sum_{\chi \in \operatorname{Irr}(G)} \frac{|G|}{\chi(1)}\left\langle N_{w} \chi, \chi\right\rangle \chi(g)
$$

Second proof. We shall make use of the following classical theorem about compact groups:

Fact (Peter-Weyl). Let $\left\{\rho_{s}\right\}$ be unitary representatives for the isomorphism classes of the irreducible representations of $G$. Let $1 \leq i, j \leq \operatorname{dim}\left(\rho_{s}\right)$ and $1 \leq k, l \leq \operatorname{dim}\left(\rho_{s^{\prime}}\right)$. Then

$$
\frac{1}{|G|} \sum_{g \in G} \rho_{s}(g)_{i, j} \overline{\rho_{s^{\prime}}(g)_{k, l}}=\frac{\delta_{s, s^{\prime}} \delta_{i, k} \delta_{j, l}}{\operatorname{dim}\left(\rho_{s}\right)}
$$

We assume $w \in \mathbf{F}_{d}=\mathbf{F}\left(x_{1}, \ldots, x_{d}\right),[w, y] \in \mathbf{F}_{d+1}=\mathbf{F}\left(x_{1}, \ldots, x_{d}, y\right)$. Note that $[w, y]$ and $[w, y]^{-1}=[y, w]$ are equivalent under $\operatorname{Aut}\left(\mathbf{F}_{d+1}\right)$, so by Proposition $1(2)$ all Fourier coefficients of $[w, y]$ are real. Let $\rho$ be a unitary representation which affords the character $\chi$. By (1.1) we 
have

$$
\begin{aligned}
N_{[w, y]}^{\chi}=\overline{N_{[w, y]}^{\chi}} & =\frac{1}{|G|} \sum_{\substack{\bar{x} \in G^{d} \\
y \in G}} \chi\left(w y w^{-1} y^{-1}\right)=\frac{1}{|G|} \sum_{\substack{\bar{x} \in G^{d} \\
y \in G}} \sum_{i=1}^{\operatorname{dim} \rho} \rho\left(w y w^{-1} y^{-1}\right)_{i, i} \\
& =\frac{1}{|G|} \sum_{\substack{\bar{x} \in G^{d} \\
y \in G}} \sum_{i, j, k, \ell=1}^{\operatorname{dim} \rho} \rho(w)_{i, j} \rho(y)_{j, k} \rho\left(w^{-1}\right)_{k, \ell} \rho\left(y^{-1}\right)_{\ell, i} \\
& =\frac{1}{|G|} \sum_{\substack{\bar{x} \in G^{d} \\
y \in G}} \sum_{i, j, k, \ell=1}^{\operatorname{dim} \rho} \rho(w)_{i, j} \rho(y)_{j, k} \overline{\rho(w)_{\ell, k} \rho(y)_{i, \ell}} \\
& \stackrel{(*)}{=} \frac{1}{\operatorname{dim} \rho} \sum_{\bar{x} \in G^{d}} \sum_{i, j, k, \ell=1}^{\operatorname{dim} \rho} \rho(w)_{i, j} \overline{\rho(w)_{\ell, k}} \delta_{i, j} \delta_{k, \ell} \\
& =\frac{1}{\operatorname{dim} \rho} \sum_{\bar{x} \in G^{d}} \sum_{i, k=1}^{\operatorname{dim} \rho} \rho(w)_{i, i} \overline{\rho(w)_{k, k}} \\
& =\frac{1}{\chi(1)} \sum_{\bar{x} \in G^{d}} \chi(w) \overline{\chi(w)} \\
& =\frac{1}{\chi(1)} \sum_{g \in G} N_{w}(g) \chi(g) \overline{\chi(g)} \\
& =\frac{|G|}{\chi(1)}\left\langle N_{w} \chi, \chi\right\rangle
\end{aligned}
$$

where $(*)$ is by Peter-Weyl.

\section{Proofs of the FORMUlas}

We begin by the formula for square letters.

Theorem 3. Let $w=w_{1} y w_{2} y w_{3} \in \mathbf{F}\left(x_{1}, \ldots, x_{d}, y\right)$ with $y$ a square, i.e. $w_{1}, w_{2}, w_{3} \in$ $\mathbf{F}\left(x_{1}, \ldots, x_{d}\right)$. Then

$$
N_{w}^{\chi}=\frac{|G|}{\chi(1)} \mathcal{F} \mathcal{S}_{\chi} N_{w_{1} w_{2}^{-1} w_{3}}^{\chi}
$$

where $\mathcal{F S}_{\chi}$ is the Frobenius-Schur indicator (1.2).

Proof. First, a word is always equivalent to its cyclic shifts under $\operatorname{Aut}\left(\mathbf{F}_{d}\right)$, so that $N_{w} \equiv$ $N_{y w_{2} y w_{3} w_{1}}$. Now applying the automorphism $x_{i} \mapsto x_{i}, y \mapsto y w_{2}^{-1}$, we obtain $N_{w} \equiv N_{y^{2} w_{2}^{-1} w_{3} w_{1}}$. Using (3.1) we have

$$
N_{w}^{\chi}=N_{y^{2} w_{2}^{-1} w_{3} w_{1}}^{\chi}=\frac{|G|}{\chi(1)} N_{y^{2}}^{\chi} N_{w_{2}^{-1} w_{3} w_{1}}^{\chi}=\frac{|G|}{\chi(1)} \mathcal{F} \mathcal{S}_{\chi} N_{w_{1} w_{2}^{-1} w_{3}}^{\chi}
$$

where we recall that $\mathcal{F} \mathcal{S}_{\chi}=N_{y^{2}}^{\chi}$ [Ser77, Proposition 39]. 
We move on to the formula for dismissible letters. We take $w \in \mathbf{F}\left(x_{1}, \ldots, x_{d}, y_{0}, \ldots, y_{n-1}\right)$ to be a word with the $y_{i}$ dismissible, and assume (by applying a cyclic shift if necessary) that $w$ ends in some $y_{i}^{ \pm}$.

Theorem 4. Let $w_{0}, \ldots, w_{2 n-1} \in F\left(x_{1}, \ldots, x_{d}\right)$, and $y_{0}, \ldots, y_{n-1}$ be new letters. Let $z_{0}, \ldots, z_{2 n-1}$ be a permutation of $y_{0}, \ldots, y_{n-1}, y_{0}^{-1}, \ldots, y_{n-1}^{-1}$, and let

$$
w=w_{0} z_{0} w_{1} z_{1} \cdots w_{2 n-1} z_{2 n-1} \text {. }
$$

Define a permutation $\tau \in \operatorname{Sym}\{0, \ldots, 2 n-1\}$ by $\tau(i)=j$ iff $z_{i}=z_{j}^{-1}$, and $\sigma \in \operatorname{Sym}\{0, \ldots, 2 n-1\}$ by $\sigma=\left(\begin{array}{lll}0 & 1 & 2 \ldots 2 n-1)\end{array} \tau\right.$ (i.e. $\left.\sigma(k)=\tau(k)+1(\bmod 2 n)\right)$. Let $\sigma=\prod_{s=1}^{r}\left(\sigma_{1}^{s} \sigma_{2}^{s} \ldots \sigma_{m_{s}}^{s}\right)$ be the decomposition of $\sigma$ into disjoint cycles. Then $r \not \equiv n(\bmod 2)$, and the Fourier expansion of $N_{w}$ is given by

$$
N_{w}^{\chi}=\frac{|G|^{n-1}}{\chi(1)^{n}} \sum_{\left(x_{1}, \ldots, x_{d}\right) \in G^{d}}\left(\prod_{i=1}^{r} \bar{\chi}(\underbrace{\prod_{j=1}^{m_{i}} w_{\sigma_{j}^{i}}}_{W_{i}})\right) .
$$

Proof. We strongly advise reading the second proof of Proposition 2 before going any further, as it is much more accessible and contains the main ideas of the proof.

First, we observe that

$$
(-1)^{r}=(-1)^{2 n-r}=\operatorname{sgn} \sigma=-\operatorname{sgn} \tau=-(-1)^{n}
$$

and thus $r \not \equiv n(\bmod 2)$. We shall write $i \boxplus j$ for $(i+j) \bmod 2 n$. We shall use the fact, already exploited in the second proof of Proposition 2, that for $\rho \in \widehat{G}$ and $1 \leq i, j, k, l \leq \operatorname{dim} \rho$,

$$
\sum_{x \in G} \rho(y)_{i, j} \rho\left(y^{-1}\right)_{k, l}=\frac{|G|}{\operatorname{dim} \rho} \delta_{i, l} \delta_{j, k},
$$

(this follows from $\rho\left(y^{-1}\right)_{k, l}=\overline{\rho(y)_{l, k}}$ and the Peter-Weyl Theorem). By (1.1) we have

$$
\begin{aligned}
& \overline{N_{w}^{\chi}(g)}=\frac{1}{|G|} \sum_{\substack{x_{1}, \ldots, x_{d} \in G \\
y_{0}, \ldots, y_{n}-1}} \chi\left(\prod_{j=0}^{2 n-1} w_{j} z_{j}\right)=\frac{1}{|G|} \sum_{\substack{x_{1}, \ldots, x_{d} \in G \\
y_{0}, \ldots, y_{n}-1 \in G}} \sum_{i=1}^{\operatorname{dim} \rho} \rho\left(\prod_{j=0}^{2 n-1} w_{j} z_{j}\right)_{i, i}= \\
& =\frac{1}{|G|} \sum_{\substack{x_{1}, \ldots, x_{d} \in G \\
y_{0}, \ldots, y_{n}-1 \in G}} \sum_{\substack{k_{0}, \ldots, k_{2 n-1}=1 \\
l_{0}, \ldots, l_{2 n-1}=1}}^{\operatorname{dim} \rho} \prod_{j=0}^{2 n-1} \rho\left(w_{j}\right)_{l_{j}, k_{j}} \prod_{j=0}^{2 n-1} \rho\left(z_{j}\right)_{k_{j}, l_{j} \boxplus 1} \\
& =\frac{|G|^{n-1}}{\chi(1)^{n}} \sum_{x_{1}, \ldots, x_{d} \in G} \sum_{\substack{k_{0}, \ldots, k_{2 n-1}=1 \\
l_{0}, \ldots, l_{2 n-1}=1}}^{\operatorname{dim} \rho} \prod_{j=0}^{2 n-1} \rho\left(w_{j}\right)_{l_{j}, k_{j}} \prod_{j=0}^{2 n-1} \delta_{k_{j}, l_{\tau(j) \boxplus 1}} \delta_{l_{j \boxplus 1}, k_{\tau(j)}} \quad \text { (by (4.1) } \\
& =\frac{|G|^{n-1}}{\chi(1)^{n}} \sum_{x_{1}, \ldots, x_{d} \in G} \sum_{\substack{k_{0}, \ldots, k_{2 n-1}=1 \\
l_{0}, \ldots, l_{2 n-1}=1}}^{\operatorname{dim} \rho} \prod_{j=0}^{2 n-1} \rho\left(w_{j}\right)_{l_{j}, k_{j}} \prod_{j=0}^{2 n-1} \delta_{k_{j}, l_{\sigma(j)}} \quad\left(\begin{array}{c}
\text { since } \tau(j) \boxplus 1=\sigma(j) \\
\text { and } \tau=\tau^{-1}
\end{array}\right) \\
& =\frac{|G|^{n-1}}{\chi(1)^{n}} \sum_{x_{1}, \ldots, x_{d} \in G} \sum_{l_{0}, \ldots, l_{2 n-1}=1}^{\operatorname{dim} \rho} \prod_{j=0}^{2 n-1} \rho\left(w_{j}\right)_{l_{j}, l_{\sigma(j)}}
\end{aligned}
$$




$$
\begin{aligned}
& =\frac{|G|^{n-1}}{\chi(1)^{n}} \sum_{x_{1}, \ldots, x_{d} \in G} \sum_{l_{0}, \ldots, l_{2 n-1}=1}^{\operatorname{dim} \rho} \prod_{s=1}^{r} \prod_{t=1}^{m_{s}} \rho\left(w_{\sigma_{t}^{s}}\right)_{l_{\sigma_{t}^{s}}, l_{\sigma\left(\sigma_{t}^{s}\right)}} \quad\left(\text { since }\left\{\sigma_{t}^{s}\right\}_{\substack{s=1 . . r \\
t=1 . . m_{s}}}=\{1 \ldots n\}\right) \\
& =\frac{|G|^{n-1}}{\chi(1)^{n}} \sum_{x_{1}, \ldots, x_{d} \in G} \sum_{l_{0}, \ldots, l_{2 n-1}}^{\operatorname{dim} \rho} \prod_{s=1}^{r} \prod_{t=1}^{m_{s}} \rho\left(w_{\sigma_{t}^{s}}\right)_{l_{\sigma_{t}^{s}}, l_{\left.\sigma_{t+1\left(\bmod m_{s}\right.}\right)}} \\
& =\frac{|G|^{n-1}}{\chi(1)^{n}} \sum_{x_{1}, \ldots, x_{d} \in G} \sum_{l_{\sigma_{0}^{1}}, l_{\sigma_{0}^{2}}, \ldots, l_{\sigma_{0}^{r}}=1}^{\operatorname{dim} \rho} \prod_{s=1}^{r} \rho\left(\prod_{t=1}^{m_{s}} w_{\sigma_{t}^{s}}\right)_{l_{\sigma_{0}^{s}}, l_{\sigma_{0}^{s}}} \\
& =\frac{|G|^{n-1}}{\chi(1)^{n}} \sum_{x_{1}, \ldots, x_{d} \in G} \prod_{s=1}^{r} \chi\left(\prod_{t=1}^{m_{s}} w_{\sigma_{t}^{s}}\right) \text {. }
\end{aligned}
$$

\section{REFERENCES}

[AV11] A. Amit and U. Vishne, Characters and solutions to equations in finite groups, Journal of Algebra and Its Applications 10 (2011), no. 4, 675-686.

[Fol95] GB Folland, A course in abstract harmonic analysis, CRC, 1995.

[Fro96] G. Frobenius, Über gruppencharaktere, Reichsdr., 1896.

[Isa94] I.M. Isaacs, Character theory of finite groups, Dover publications, 1994.

[Ser77] J.P. Serre, Linear representations of finite groups, vol. 42, Springer Verlag, 1977.

[Tam00] T. Tambour, The number of solutions of some equations in finite groups and a new proof of itô's theorem, Communications in Algebra 28 (2000), no. 11, 5353-5361.

Einstein Institute of Mathematics, Hebrew University, Jerusalem

E-mail address: parzan@math.huji.ac.il, gili.schul@mail.huji.ac.il 